implicated in activation of fibroblasts and could contribute to fibrogenic responses in lung disease. However, further studies are required to confirm relative importance within the family and reveal mechanisms of action.

\section{S121 INDIVIDUAL CELL TRACKING IN A TRANSGENIC ZEBRAFISH INFLAMMATION MODEL REVEALS THE FATES OF INFLAMMATORY NEUTROPHILS DURING INFLAMMATION RESOLUTION}

doi:10.1136/thx.2010.150946.22

${ }^{1} \mathrm{G}$ Dixon, ${ }^{1} \mathrm{C}$ A Loynes, ${ }^{2} \mathrm{X}$ Wang, ${ }^{2} \mathrm{P}$ W Ingham, ${ }^{1} \mathrm{M} \mathrm{K}$ B Whyte, ${ }^{1} \mathrm{~S}$ A Renshaw. ${ }^{1} \mathrm{MRC}$ Centre for Developmental and Biomedical Genetics, Sheffield, UK; ${ }^{2} I M C B,{ }^{*} S T A R$, Singapore, Singapore

Removal of inflammatory neutrophils from sites of inflammation can occur by a number of routes; into exudates, by apoptosis followed by macrophage clearance and by retrograde chemotaxis. The relative contribution of these disposal mechanisms in vivo has been hard to define, and the lifespan of an in vivo tissue neutrophil has been hard to directly measure. We have generated transgenic zebrafish expressing the fluorescent photo-convertible protein, Kaede, in neutrophils.

Objective To label individual inflammatory neutrophils and track their fate during inflammation resolution in vivo.

Method Individual neutrophils were marked by photoconverting the Kaede protein using $405 \mathrm{~nm}$ laser light restricted to the individual cell profiles. Known numbers of neutrophils were photoconverted and visualised over $48 \mathrm{~h}$. In subsequent experiments, an inflammatory reaction was induced by sterile tail transection of transparent zebrafish larvae. Kaede labelled neutrophils are recruited to the site of injury where they can be photoconverted and followed using time lapse video microscopy.

Results By counting the number of remaining photoconverted neutrophils over time, the half-life of a neutrophil was calculated. Our data suggest the lifespan of a zebrafish neutrophil in the tissues is 117.7 (CI 95.67 to 157.8) h, a figure comparable to that inferred for human tissue neutrophils. Timelapse videos reveal a population of neutrophils that migrate away from the site of injury, undergoing retrograde chemotaxis. Whilst neutrophils can migrate away from the site of injury, they are not completely free to do so. The apparent restriction on their behaviour may be due to the presence of a persisting chemical gradient or may reflect an intrinsic feature of neutrophil behaviour.

Conclusions These data demonstrate the power of this model to inform our understanding of phagocyte behaviour and interaction in vivo.

\section{S122 THE M1 MACROPHAGE PHENOTYPE ACCENTUATES TGF- $\beta 1$ DRIVEN EPITHELIAL TO MESENCHYMAL TRANSITION (EMT) VIA THE SECRETION OF TNF $\alpha$}

doi:10.1136/thx.2010.150946.23

L A Borthwick, M Brodlie, G E Johnson, C Ward, J Perry, P A Corris, A De-Soyza, A J Fisher. Newcastle University, Newcastle-Upon-Tyne, UK

Introduction Obliterative Bronchiolitis (OB) is characterised by fibrotic obliteration of small airways which adversely affecting graft function and survival after lung transplantation. It has been shown in vitro and in vivo that primary bronchial epithelial cells (PBEC) from the transplanted lung can undergo epithelial to mesenchymal transition (EMT) and this process may contribute to the development of $\mathrm{OB}$. We have shown that activated macrophages can disrupt epithelial wound repair by accentuating TGF- $\beta 1$-driven EMT. We hypothesised that this effect might be limited to macrophages with an $\mathrm{M} 1$ phenotype and that their secretory products might be a target for limiting the inflammatory accentuation of EMT.

Methods and materials The THP-1 monocytic cell line was stimulated with clinical isolates of Pseudomonas aeruginosa (PA) and the effect of the activated cells or conditioned media on TGF- $\beta 1$-driven EMT assessed in PBEC (Western blotting, confocal microscopy). In addition, THP-1 cells were differentiated to an M1 phenotype by treatment with IFN $\gamma$ and an M2 phenotype with IL-4/IL-13 and cytokine release (ELISA) and their effect on TGF- $\beta 1$ driven EMT assessed. The effect of blocking TNF $\alpha$ secreted from activated THP1 cells on EMT was assessed using an anti-TNF $\alpha$ antibody.

Results Treatment with TGF- $\beta 1+$ activated THP-1 cells had no effect on EMT marker expression $(p>0.05 n=6)$. However, cotreatment with TGF- $\beta 1+$ conditioned media from activated THP-1 cells dramatically accentuated TGF- $\beta 1$-driven EMT $(p<0.05 n=6)$ M1 differentiated THP-1 cells released 8.4-fold more TNF $\alpha$ and 8.1fold more IL- $1 \beta$ than $M 2$ cells $(p<0.05, n=3)$. Conditioned media from $M 1$, but not $M 2$, cells dramatically accentuated TGF- $\beta 1$ driven EMT $(p<0.05 n=6)$. Blocking TNF $\alpha$ in the conditioned media from THP-1 cells significantly inhibits the decrease in E-cadherin $(39 \% \pm$ $4 \%)$ and the increase in vimentin $(59 \% \pm 18 \%)$ and fibronectin $(72 \% \pm 14 \%)$ expression $(\mathrm{p}<0.05, \mathrm{n}=5)$.

Conclusion The secretory products of $\mathrm{M} 1$, but not $\mathrm{M} 2$, macrophages significantly accentuate TGF- $\beta 1$ driven EMT. TNF $\alpha$ appears to be a major constituent of this accentuating action. This raises the possibility that either TNF $\alpha$ targeted therapies or modulation of macrophage phenotype may inhibit the inflammatory accentuation of EMT in airway epithelium.

\section{S123 MONONUCLEAR INFLAMMATION AND DISRUPTION OF NORMAL ALVEOLAR STRUCTURE FOLLOWING DELETION OF G $\alpha 0 / 11$, BUT NOT G $\alpha 12 / 13$, IN TYPE II ALVEOLAR EPITHELIAL CELLS}

doi:10.1136/thx.2010.150946.24

A E John, J Porte, L Jolly, A L Tatler, R G Jenkins. University of Nottingham, Nottingham, UK

Activation of latent TGF $\beta$ by the epithelially restricted $\alpha_{v} \beta 6$ integrin is induced by activators of the RhoA signalling pathway and is critical in the pathogenesis of lung injury and fibrosis. The G-proteins, Ga12 and Ga13 are known to activate RhoA and we have previously shown that the $\alpha_{\mathrm{v}} \beta 6$ integrin can mediate TGF $\beta$ activation via Gaq and RhoA. To establish the role of these Gproteins in both normal lung development and following lung injury, we generated mice with a targeted deletion of $\mathrm{G} \alpha \mathrm{q} / 11$ or G $\alpha 12 / 13$ in SpC-positive Type II alveolar epithelial cells. SpC-Cre mice were crossed with either Gaq(flox-flox)/11(-/-) or Ga12(-/-)/13 (flox-flox) mice and the lungs analysed histologically at 6 and 8 weeks after birth. At 6 weeks, lungs from mice with a homozygous deficiency in SpC-Gaq/11 contained focal inflammatory infiltrates consisting primarily of mononuclear leukocytes. Inflammation was associated with the localised disruption of normal alveolar architecture and the appearance of abnormal Type I and Type II alveolar epithelial cells, identified by SpC and T1 $\alpha$ immunohistochemistry, within in the alveolar airspaces. Furthermore, immunohistochemical analysis of phosho-Smad2 levels in these lungs detected increased staining in the inflammatory foci within the homozygous SpC-G $\alpha q / 11$ knockout lungs. At 8 weeks, the inflammatory foci were more numerous and lung architecture was severely disrupted with multiple abnormally large alveolar airspaces detected. In contrast, mice with at least one floxed Gaq or null Ga11 allele showed no abnormalities at either 6 or 8 weeks. We also detected no abnormal lung phenotype in 6- and 8-week old mice with a homozygous or heterozygous deficiency in SpC-G $\alpha 12 / 13$. These data suggest that Ga11/q signalling is required to prevent 